\title{
ASYMPTOTIC EXPANSION FOR TRANSPORT PROCESSES IN SEMI-MARKOV MEDIA
}

UDC 519.21

\section{A. A. POGORUI AND RAMÓN M. RODRÍGUEZ-DAGNINO}

\begin{abstract}
In this paper we study asymptotic expansions for a solution of the singularly perturbed equation for a functional of a semi-Markov random evolution on the line. By using the method for solutions of singularly perturbed equations, we obtain the solution in the form of a series of regular and singular terms. The first regular term satisfies a diffusion-type equation, and the first singular term is a semigroup with the infinitesimal operator of the respective related bivariate process. Each regular and singular term can be calculated recursively.
\end{abstract}

\section{INTRODUCTION}

Asymptotic expansions for perturbed equations of Markov and semi-Markov random evolution have generated a great deal of research; see [7]-[10] and others. In this paper we investigate solutions of singularly perturbed equations of semi-Markov random evolutions by reducing a semi-Markov process to a Markov process with a more complicated phase space.

Let $\{\xi(t), t \geq 0\}$ be a semi-Markov process on the phase space $\{\mathbb{E}, \mathcal{F}\}$ with the semiMarkov kernel

$$
Q=(x, B, t)=P(x, B) G_{x}(t), \quad B \in \mathcal{F},
$$

where $P(x, B)$ are the transition probabilities of the embedded Markov chain $\left\{\xi_{n}, n \geq 0\right\}$, and $G_{x}(t)$ is the cumulative distribution function (cdf) of a sojourn time (holding time) of $\xi(t)$ in $x \in \mathbb{E}$.

Now, let us assume a function $\mathcal{C}(u, x), u \in \mathbb{R}, x \in \mathbb{E}$ such that it satisfies the uniquevalued solvability condition of the following evolution equation:

$$
\frac{d u(t, x)}{d t}=\mathcal{C}(u(t, x), x), \quad u(0, x)=u_{0} .
$$

In addition, we assume that the derivative $\partial \mathcal{C}(u, x) / \partial u$ is bounded.

For the fixed parameter $\varepsilon>0$ consider the following random transport process $u_{\varepsilon}(t)$ in the scaled semi-Markov medium $\left\{\xi\left(t / \varepsilon^{2}\right)\right\}$, as follows [1, 2]:

$$
\frac{d u_{\varepsilon}(t)}{d t}=\frac{1}{\varepsilon} \mathcal{C}\left(u_{\varepsilon}(t), \xi\left(t / \varepsilon^{2}\right)\right), \quad u_{\varepsilon}(0)=u_{0}
$$

2010 Mathematics Subject Classification. Primary 60J25; Secondary 35C20.

Key words and phrases. Asymptotic expansion, semi-Markov, random evolution, singular perturbed equation.

We thank ITESM, Campus Monterrey, through the Research Chair in Telecommunications, for the support provided in the development of this work. 
Let us assume that the following four conditions hold:

C1. There exist the probability density function (pdf) $g_{x}(t)=\frac{d}{d t} G_{x}(t)$ and the first two moments $m_{x}^{(1)}=\int_{0}^{\infty} t g_{x}(t) d t$ and $m_{x}^{(2)}=\int_{0}^{\infty} t^{2} g_{x}(t) d t$ for all $x \in \mathbb{E}$.

C2. The embedded Markov chain $\xi_{n}$ is uniformly ergodic with the stationary distribution

$$
\rho(A)=\int_{\mathbb{E}} \rho(d x) P(x, A), \quad A \in \mathcal{F} .
$$

C3. The following supremum is bounded:

$$
\sup _{x, u} \frac{\int_{u}^{\infty} \bar{G}_{x}(t) d t}{\bar{G}_{x}(u)}<\infty
$$

where $\bar{G}_{x}(u)=1-G_{x}(u)$ is the survival function of the sojourn time in state $x$.

C4. The following moments are positive:

$$
\widehat{m}^{(k)}=\int_{\mathbb{X}} \rho(d x) m_{x}^{(k)}>0, \quad k=1,2 .
$$

\section{Asymptotic expansion of the RANDOM SWitching PRocess}

Denote by $\mathbb{X}=\mathbb{E} \times[0, \infty)$ and by $\mathcal{X}=\mathcal{F} \times \mathcal{B}_{+}$, where $\mathcal{B}_{+}$is the Borel $\sigma$-algebra on $[0, \infty)$.

On the phase space $\mathbb{X}$ we consider the following bivariate process:

$$
\{\varsigma(t)=(\xi(t), \tau(t)), t \geq 0\}
$$

where $\tau(t)=t-\sup \left\{u \leq t: \xi_{\varepsilon}(u) \neq \xi_{\varepsilon}(t)\right\}$.

It is well known that $\varsigma(t)$ is Markovian and its infinitesimal operator $Q$ can be written as $([3]-[\underline{5})$

where

$$
Q \varphi(x, \tau)=r_{x}(\tau)[P \varphi(x, 0)-\varphi(x, \tau)]+\frac{\partial}{\partial \tau} \varphi(x, \tau)
$$

$$
\begin{gathered}
r_{x}(t)=\frac{g_{x}(t)}{1-G_{x}(t)}, \\
P \varphi(x, 0)=\int_{\mathbb{E}} P(x, d y) \varphi(y, 0),
\end{gathered}
$$

and $\varphi(x, \tau)$ is a continuously differentiable function with respect to $\tau$.

We denote by $\mathbf{B}$ the Banach space of bounded $\mathcal{F}$-measurable functions on $\mathbb{X}$ with supremum norm.

Let us introduce the operator $\Pi_{1} f=(\pi, f) \mathbb{I}(x, \tau)$, where $f \in \mathbf{B}$, and $\mathbb{I}(x, \tau)=1$, for all $(x, \tau) \in \mathbb{X}$

$$
\pi(B \times[0, s])=\int_{B} \int_{0}^{s} \rho(d x) \bar{G}_{x}(s) d s / \widehat{m}^{(1)},
$$

and the inner product

$$
(\pi, f)=\int_{\mathbb{X}} \pi(d z) f(z)=\int_{\mathbb{X}} \int_{0}^{\infty} \rho(d x) \bar{G}_{x}(s) f(x, s) d s / \widehat{m}^{(1)} .
$$

It is well known that $\Pi_{1}$ is the projection operator on $\operatorname{ker}(Q)$, i.e., $\Pi_{1} Q=Q \Pi_{1}=0$; see p. 139 in [4].

Now, denote as $R_{0}$ the potential operator of the embedded Markov chain $\xi_{n}$, i.e., $R_{0}=\Pi_{0}-\left(P-\Pi_{0}\right)^{-1}$, where (see [5])

$$
\Pi_{0} g=\int_{\mathbb{E}} \rho(d x) g(x) \mathbb{I}(x, \tau) .
$$


In Lemma 2.2 of [4] it is proved that under conditions C1-C4 the potential operator $\mathcal{R}_{1}$ of $\varsigma(t)$ is given by

$$
\begin{aligned}
\mathcal{R}_{1} f(x, u)= & \int_{0}^{\infty} \frac{\bar{G}_{x}(t)}{\bar{G}_{x}(u)} f(x, t) d t-(\pi, f) \int_{0}^{\infty} \frac{\bar{G}_{x}(t)}{\bar{G}_{x}(u)} d t \\
& -\frac{1}{\widehat{m}^{(1)}} \int_{\mathbb{X}} \int_{y=0}^{\infty} \int_{z=y}^{\infty} \rho(d x) \bar{G}_{x}(s) f(x, z) d y d z+\frac{\widehat{m}^{(2)}}{2 \widehat{m}^{1}}(\pi, f) \\
& +\left(I-\Pi_{1}\right) P R_{0} \int_{0}^{\infty} \bar{G}_{x}(z) f(x, z) d z-(\pi, f)\left(I-\Pi_{1}\right) P R_{0} \widehat{m}^{1}
\end{aligned}
$$

We should recall that the potential operator of $\varsigma(t)$ is the generalized inverse operator of $Q$ and it satisfies $\mathcal{R}_{1} Q=Q \mathcal{R}_{1}=I-\Pi_{1}$.

It is also well known that the three-component process $\zeta(t)=(u(t, x), \xi(t), \tau(t))$ is Markovian on the phase space $\mathbb{R} \times \mathbb{E} \times[0, \infty)$ with the infinitesimal operator $A$ given by [1, 2, 4]:

$$
\begin{aligned}
A \varphi(u, x, \tau) & =\mathcal{C}(u, x) \frac{\partial}{\partial u} \varphi(u, x, \tau)+Q \varphi(u, x, \tau) \\
& =\mathcal{C}(u, x) \frac{\partial}{\partial u} \varphi(u, x, \tau)+r_{x}(\tau)[P \varphi(u, x, 0)-\varphi(u, x, \tau)]+\frac{\partial}{\partial \tau} \varphi(u, x, \tau),
\end{aligned}
$$

where $\varphi(u, z, \tau)$ is a continuously differentiable function with respect to $u$ and $\tau$ and it is in the domain of the operator $A$, and

$$
P \varphi(u, z, 0)=\int_{\mathbb{E}} P(z, d y) \varphi(u, y, 0) .
$$

Let us consider the scaled process $\zeta_{\varepsilon}(t)=\left(u_{\varepsilon}(t, x), \xi_{\varepsilon}\left(t / \varepsilon^{2}\right), \tau\left(t / \varepsilon^{2}\right)\right)$. It can be easily verified that its infinitesimal operator $A_{\varepsilon}$ satisfies

$$
\begin{aligned}
A_{\varepsilon} \varphi(u, x, \tau)= & \frac{1}{\varepsilon} \mathcal{C}(u, x) \frac{\partial}{\partial u} \varphi(u, x, \tau)+\frac{1}{\varepsilon^{2}} r_{x}(\tau)[P \varphi(u, x, 0)-\varphi(u, x, \tau)] \\
& +\frac{1}{\varepsilon^{2}} \frac{\partial}{\partial \tau} \varphi(u, x, \tau) .
\end{aligned}
$$

By defining $\varphi_{\varepsilon}(t, u, x, \tau)=\mathrm{E}\left[\varphi\left(\zeta_{\varepsilon}(t)\right) / \zeta_{\varepsilon}(0)=(u, x, \tau)\right]$ we can write the inverse Kolmogorov equation for $\zeta_{\varepsilon}(t)$ as follows:

$$
\begin{aligned}
\frac{\partial}{\partial t} \varphi_{\varepsilon}(t, u, x, \tau)= & A_{\varepsilon} \varphi_{\varepsilon}(t, u, x, \tau) \\
= & \frac{1}{\varepsilon} \mathcal{C}(u, x) \frac{\partial}{\partial u}, \varphi_{\varepsilon}(t, u, x, \tau) \\
& +\frac{1}{\varepsilon^{2}} r_{x}(\tau)\left[P \varphi_{\varepsilon}(t, u, x, 0)-\varphi_{\varepsilon}(t, u, x, \tau)\right]+\frac{1}{\varepsilon^{2}} \frac{\partial}{\partial \tau} \varphi_{\varepsilon}(t, u, x, \tau),
\end{aligned}
$$

with the boundary condition $\varphi_{\varepsilon}(0, u, x, \tau)=\varphi_{\varepsilon}^{(0)}$.

Theorem. Suppose that conditions $C 1$ to $C_{4}$ are fulfilled. In addition, assume that the following balance condition holds:

$$
\Pi_{1} \mathcal{C}(u, x) \Pi_{1}=0 .
$$

Then, the solution of (3) can be expanded in the following form:

$$
\varphi_{\varepsilon}(t, u, x, \tau)=\varphi^{(0)}(t, u, x, \tau)+\sum_{n=1}^{\infty} \varepsilon^{n}\left(\varphi^{(n)}(t, u, x, \tau)+\psi^{(n)}\left(\frac{t}{\varepsilon^{2}}, u, x, \tau\right)\right),
$$


where the first regular term $\varphi^{(0)}(t, u, x, \tau)$ satisfies the diffusion equation

$$
\frac{\partial}{\partial t} \varphi^{(0)}(t, u, x, \tau)+\Pi_{1} \mathcal{C}(u, x) \frac{\partial}{\partial u} \mathcal{R}_{o} \mathcal{C}(u, x) \frac{\partial}{\partial u} \Pi_{1} \varphi^{(0)}(t, u, x, \tau)=0
$$

and all the terms $\varphi^{(k)}(t, u, x, \tau), k \geq 1$, can be calculated in a recursive manner. The first singular term is of the following form: $\psi^{(1)}(t, u, x, \tau)=\psi^{(1)}(0, u, x, \tau) \exp \{Q t\}$, and all the terms $\psi^{(k)}(t, u, x, \tau), k \geq 2$, can be recursively calculated in the following form:

$$
\begin{aligned}
\psi^{(k+1)}(t, u, x, \tau)= & \psi^{(k+1)}(0, u, x, \tau) \exp \{Q t\} \\
& +\int_{0}^{t} \exp \{Q(t-s)\} \mathcal{C}(u, x) \frac{\partial}{\partial u} \psi^{(k)}(s, u, x, \tau) d s .
\end{aligned}
$$

Proof. By applying the method for singularly perturbed equations considered in [6] we can find a solution of (3) in the following form:

$$
\begin{gathered}
\varphi_{\varepsilon}(t, u, x, \tau)=\varphi^{(0)}(t, u, x, \tau)+\sum_{n=1}^{\infty} \varepsilon^{n}\left(\varphi^{(n)}(t, u, x, \tau)+\psi^{(n)}\left(\frac{t}{\varepsilon^{2}}, u, x, \tau\right)\right), \\
\varphi_{\varepsilon}^{(0)}=\varphi^{(0)}(0, u, x, \tau)+\sum_{n=1}^{\infty} \varepsilon^{n}\left(\varphi^{(n)}(0, u, x, \tau)+\psi^{(n)}(0, u, x, \tau)\right),
\end{gathered}
$$

where $\varphi^{(n)}(t, u, x, \tau), n \geq 0$, are regular terms and $\psi^{(n)}\left(t / \varepsilon^{2}, u, x, \tau\right), n \geq 1$, are singular terms of the expansion (4).

Substituting (44) into (3), we have for regular terms $\varphi^{(0)}(t, u, x, \tau)$,

$$
Q \varphi^{(0)}(t, u, x, \tau)=r_{x}(\tau)\left[P \varphi^{(0)}(t, u, x, 0)-\varphi^{(0)}(t, u, x, \tau)\right]+\frac{\partial}{\partial \tau} \varphi^{(0)}(t, u, x, \tau)=0 .
$$

Therefore $\varphi^{(0)}(t, u, x, \tau) \in \operatorname{ker}(Q)$.

Then, we can obtain for $\varphi^{(1)}(t, u, x, \tau)$,

$$
\begin{aligned}
\mathcal{C}(u, x) & \frac{\partial}{\partial u} \varphi^{(0)}(t, u, x, \tau)+r_{x}(\tau)\left[P \varphi^{(1)}(t, u, x, 0)-\varphi^{(1)}(t, u, x, \tau)\right] \\
+\frac{\partial}{\partial \tau} \varphi^{(1)}(t, u, x, \tau) & =0 .
\end{aligned}
$$

Hence,

$$
\varphi^{(1)}(t, u, x, \tau)=-\mathcal{R}_{1} \mathcal{C}(u, x) \frac{\partial}{\partial u} \varphi^{(0)}(t, u, x, \tau)+n_{1}(t, u, x, \tau)
$$

where $n_{1}(t, u, x, \tau) \in \operatorname{ker}(Q)$ and it depends on the initial conditions of equation (4).

Now, for $k \geq 2$ we have

$$
\begin{aligned}
\frac{\partial}{\partial t} \varphi^{(k-2)}(t, u, x, \tau)= & \mathcal{C}(u, x) \frac{\partial}{\partial u} \varphi^{(k-1)}(t, u, x, \tau) \\
& +r_{x}(\tau)\left[P \varphi^{(k)}(t, u, x, 0)-\varphi^{(k)}(t, u, x, \tau)\right]+\frac{\partial}{\partial \tau} \varphi^{(k)}(t, u, x, \tau) .
\end{aligned}
$$

Hence,

$$
Q \varphi^{(k)}(t, u, x, \tau)=\frac{\partial}{\partial t} \varphi^{(k-2)}(t, u, x, \tau)-\mathcal{C}(u, x) \frac{\partial}{\partial u} \varphi^{(k-1)}(t, u, x, \tau) .
$$


For the specific $k=2$ case, we have

$$
\begin{aligned}
Q \varphi^{(2)}(t, u, x, \tau)= & \frac{\partial}{\partial t} \varphi^{(0)}(t, u, x, \tau)-\mathcal{C}(u, x) \frac{\partial}{\partial u} \varphi^{(1)}(t, u, x, \tau) \\
= & \frac{\partial}{\partial t} \varphi^{(0)}(t, u, x, \tau)+\mathcal{C}(u, x) \frac{\partial}{\partial u} \mathcal{R}_{1} \mathcal{C}(u, x) \frac{\partial}{\partial u} \varphi^{(0)}(t, u, x, \tau) \\
& -\mathcal{C}(u, x) \frac{\partial}{\partial u} n_{1}(t, u, x, \tau) .
\end{aligned}
$$

Now, by applying the operator $\Pi_{1}$ on the left,

$$
\Pi_{1} Q \varphi^{(2)}(t, u, x, \tau)=0=\frac{\partial}{\partial t} \Pi_{1} \varphi^{(0)}(t, u, x, \tau)-\Pi_{1} \mathcal{C}(u, x) \frac{\partial}{\partial u} \varphi^{(1)}(t, u, x, \tau),
$$

and taking into account that $\varphi^{(0)}(t, u, x, \tau) \in \operatorname{ker}(Q)$, i.e.,

$$
\Pi_{1} \varphi^{(0)}(t, u, x, \tau)=\varphi^{(0)}(t, u, x, \tau),
$$

we obtain

$$
\begin{aligned}
0= & \frac{\partial}{\partial t} \varphi^{(0)}(t, u, x, \tau)+\Pi_{1} \mathcal{C}(u, x) \frac{\partial}{\partial u} \mathcal{R}_{1} \mathcal{C}(u, x) \frac{\partial}{\partial u} \Pi_{1} \varphi^{(0)}(t, u, x, \tau) \\
& -\Pi_{1} \mathcal{C}(u, x) \frac{\partial}{\partial u} \Pi_{1} n_{1}(t, u, x, \tau)
\end{aligned}
$$

Since the operator $\Pi_{1}$ does not depend on $u$, then $\Pi_{1} \mathcal{C}(u, x) \frac{\partial}{\partial u} \Pi_{1} n_{1}(t, u, x, \tau)$ is equivalent to

$$
\Pi_{1} \mathcal{C}(u, x) \Pi_{1} \frac{\partial}{\partial u} n_{1}(t, u, x, \tau)=0,
$$

because of the balance condition $\Pi_{1} \mathcal{C}(u, x) \Pi_{1}=0$.

Therefore,

$$
\frac{\partial}{\partial t} \varphi^{(0)}(t, u, x, \tau)+\Pi_{1} \mathcal{C}(u, x) \frac{\partial}{\partial u} \mathcal{R}_{1} \mathcal{C}(u, x) \frac{\partial}{\partial u} \Pi_{1} \varphi^{(0)}(t, u, x, \tau)=0 .
$$

Since $\mathcal{R}_{1}$ does not operate on $u$ it is easy to see that (8) is the diffusion equation and therefore $\varphi^{(0)}(t, u, x, \tau)$ is a solution of this diffusion equation.

Similarly, it follows from (7) that

$$
\varphi^{(k)}(t, u, x, \tau)=\mathcal{R}_{1}\left[\frac{\partial}{\partial t} \varphi^{(k-2)}(t, u, x, \tau)-\mathcal{C}(u, x) \frac{\partial}{\partial u} \varphi^{(k-1)}(t, u, x, \tau)\right]+n_{k}(t, x, u, \tau),
$$

where $n_{k}(t, x, u, \tau) \in \operatorname{ker}(Q)$.

To find $n_{k}(t, u, x, \tau)$ we use the fact that $\varphi^{(0)}(t, u, x, \tau) \in \operatorname{ker}(Q)$, and we put

$$
n_{0}(t, x, u, \tau)=\varphi^{(0)}(t, u, x, \tau) .
$$

From (6) and (9) we have for $k=2$,

$$
\begin{aligned}
\varphi^{(2)}(t, u, x, \tau)= & \mathcal{R}_{1} \frac{\partial}{\partial t} n_{0}(t, u, x, \tau)+\mathcal{R}_{1} \mathcal{C}(u, x) \frac{\partial}{\partial u} \mathcal{R}_{1} \mathcal{C}(u, x) \frac{\partial}{\partial u} n_{0}(t, u, x, \tau) \\
& -\mathcal{R}_{1} \mathcal{C}(u, x) \frac{\partial}{\partial u} n_{1}(t, u, x, \tau)+n_{2}(t, u, x, \tau)
\end{aligned}
$$

By letting $k=3$ in (7) we obtain

$$
Q \varphi^{(3)}(t, u, x, \tau)=\frac{\partial}{\partial t} \varphi^{(1)}(t, u, x, \tau)-\mathcal{C}(u, x) \frac{\partial}{\partial u} \varphi^{(2)}(t, u, x, \tau) .
$$


Then by using (6) and (10) it follows that

$$
\begin{aligned}
Q \varphi^{(3)}(t, u, x, \tau)= & \frac{\partial}{\partial t} \varphi^{(1)}(t, u, x, \tau)-\mathcal{C}(u, x) \frac{\partial}{\partial u} \varphi^{(2)}(t, u, x, \tau) \\
= & -\frac{\partial}{\partial t} \mathcal{R}_{1} \mathcal{C}(u, x) \frac{\partial}{\partial u} n_{0}(t, u, x, \tau)+\frac{\partial}{\partial t} n_{1}(t, u, x, \tau) \\
& -\mathcal{C}(u, x) \frac{\partial}{\partial u} \mathcal{R}_{1} \frac{\partial}{\partial t} n_{0}(t, u, x, \tau) \\
& -\mathcal{C}(u, x) \frac{\partial}{\partial u} \mathcal{R}_{1} \mathcal{C}(u, x) \frac{\partial}{\partial u} \mathcal{R}_{1} \mathcal{C}(u, x) \frac{\partial}{\partial u} n_{0}(t, u, x, \tau) \\
& +\mathcal{C}(u, x) \frac{\partial}{\partial u} \mathcal{R}_{1} \mathcal{C}(u, x) \frac{\partial}{\partial u} n_{1}(t, u, x, \tau)-\mathcal{C}(u, x) \frac{\partial}{\partial u} n_{2}(t, u, x, \tau) .
\end{aligned}
$$

Taking into account the balance condition $\Pi_{1} \mathcal{C}(u, x) \Pi_{1}=0$ and multiplying (11) by $\Pi_{1}$, we have

$$
\begin{gathered}
0=\left(\frac{\partial}{\partial t}+\Pi_{1} \mathcal{C}(u, x) \frac{\partial}{\partial u} \mathcal{R}_{1} \mathcal{C}(u, x) \Pi_{1} \frac{\partial}{\partial u}\right) n_{1}(t, u, x, \tau) \\
-\left(\Pi_{1} \mathcal{C}(u, x) \frac{\partial}{\partial u} \mathcal{R}_{1} \frac{\partial}{\partial t} \Pi_{1}+\Pi_{1} \frac{\partial}{\partial t} \mathcal{R}_{1} \mathcal{C}(u, x) \Pi_{1} \frac{\partial}{\partial u}\right. \\
\left.\quad+\Pi_{1} \mathcal{C}(u, x) \frac{\partial}{\partial u} \mathcal{R}_{1} \mathcal{C}(u, x) \frac{\partial}{\partial u} \mathcal{R}_{1} \mathcal{C}(u, x) \Pi_{1} \frac{\partial}{\partial u}\right) n_{0}(t, u, x, \tau)
\end{gathered}
$$

After solving (12), we can express $n_{1}(t, u, x, \tau)$ in terms of $n_{0}(t, u, x, \tau)$.

Now, we consider (77) for $k=4$, namely,

$$
Q \varphi^{(4)}(t, u, x, \tau)=\frac{\partial}{\partial t} \varphi^{(2)}(t, u, x, \tau)-\mathcal{C}(u, x) \frac{\partial}{\partial u} \varphi^{(3)}(t, u, x, \tau) .
$$

Let us substitute

$$
Q \varphi^{(3)}(t, u, x, \tau)=\mathcal{R}_{1}\left[\frac{\partial}{\partial t} \varphi^{(1)}(t, u, x, \tau)-\mathcal{C}(u, x) \frac{\partial}{\partial u} \varphi^{(2)}(t, u, x, \tau)\right]+n_{3}(t, u, x, \tau)
$$

and $\varphi^{(2)}(t, u, x, \tau)$ from (10) into (13), and then we multiply by $\Pi_{1}$ both sides of the resulting equation.

In the same manner we use the balance condition stated in this theorem. Then we obtain a differential equation that relates $n_{1}(t, u, x, \tau)$ and $n_{2}(t, u, x, \tau)$. After solving this equation we can express $n_{2}(t, u, x, \tau)$ in terms of $n_{1}(t, u, x, \tau)$. The same procedure can be applied to obtain $n_{i}(t, u, x, \tau)$ for all $i=0,1,2, \ldots$ as follows:

$$
\begin{aligned}
& 0=\left(\frac{\partial}{\partial t}+\Pi_{1} \mathcal{C}(u, x) \frac{\partial}{\partial u} \mathcal{R}_{1} \mathcal{C}(u, x) \Pi_{1} \frac{\partial}{\partial u}\right) n_{i+1}(t, u, x, \tau) \\
&-\left(\Pi_{1} \mathcal{C}(u, x) \frac{\partial}{\partial u} \mathcal{R}_{1} \frac{\partial}{\partial t} \Pi_{1}+\Pi_{1} \frac{\partial}{\partial t} \mathcal{R}_{1} \mathcal{C}(u, x) \Pi_{1} \frac{\partial}{\partial u}\right. \\
&\left.\quad+\Pi_{1} \mathcal{C}(u, x) \frac{\partial}{\partial u} \mathcal{R}_{1} \mathcal{C}(u, x) \frac{\partial}{\partial u} \mathcal{R}_{1} \mathcal{C}(u, x) \Pi_{1} \frac{\partial}{\partial u}\right) n_{i}(t, u, x, \tau)
\end{aligned}
$$

Taking into account (9) and (14), we get $\varphi^{(k)}(t, u, x, \tau)$ for all $k=0,1,2, \ldots$.

Then, regarding the first singular term we have

$$
\frac{\partial}{\partial t} \psi^{(1)}=Q \psi^{(1)}
$$

and for $k \geq 1$, we have

$$
\frac{\partial}{\partial t} \psi^{(k+1)}-Q \psi^{(k+1)}=\mathcal{C}(u, x) \frac{\partial}{\partial u} \psi^{(k)} .
$$


By solving equation (15), we get

$$
\psi^{(1)}(t, u, x, \tau)=\exp _{0}\{Q t\} \psi^{1}(0, u, x, \tau),
$$

where $\exp _{0}\{Q t\}$ is a modified exponent of the following form: $\exp _{0}\{Q t\}=\exp \{Q t\}-\Pi_{1}$. For this case we have $\lim _{t \rightarrow \infty} \psi^{(1)}(t, u, x, \tau)=0$.

Taking into account (15) we obtain

$$
\begin{aligned}
\psi^{(k+1)}(t, u, x, \tau)= & \exp _{0}\{Q t\} \psi^{(k+1)}(0, u, x, \tau) \\
& +\int_{0}^{t} \exp _{0}\{Q(t-s)\} \mathcal{C}(u, x) \frac{\partial}{\partial u} \psi^{(k)}(s, u, x, \tau) d s .
\end{aligned}
$$

Thus, $\psi^{(k)}$ can be obtained from (16) recursively for all $k \geq 1$.

Therefore, in the expansion (44) the coefficient of $\varepsilon^{k}$ for $k \geq 2$ is of the following form:

$$
\begin{aligned}
\varphi^{(k)}(t, & u, x, \tau)+\psi^{(k)}\left(\frac{t}{\varepsilon^{2}}, u, x, \tau\right) \\
= & \mathcal{R}_{1}\left[\frac{\partial}{\partial t} \varphi^{(k-2)}(t, u, x, \tau)-\mathcal{C}(u, x) \frac{\partial}{\partial u} \varphi^{(k-1)}(t, u, x, \tau)\right]+n_{k}(t, u, x, \tau) \\
& +\exp _{0}\left\{Q t / \varepsilon^{2}\right\} \psi^{(k)}(0, u, x, \tau) \\
& +\int_{0}^{t / \varepsilon^{2}} \exp _{0}\left\{Q\left(t / \varepsilon^{2}-s\right)\right\} \mathcal{C}(u, x) \frac{\partial}{\partial u} \psi^{(k-1)}(s, u, x, \tau) d s .
\end{aligned}
$$

The coefficient of $\varepsilon$ is as follows:

$$
\begin{aligned}
& \varphi^{(1)}(t, u, x, \tau)+\psi^{(1)}\left(\frac{t}{\varepsilon^{2}}, u, x, \tau\right) \\
& \quad=-\mathcal{R}_{1} \mathcal{C}(u, x) \frac{\partial}{\partial u} \varphi^{(0)}(t, u, x, \tau)+n_{1}(t, u, x, \tau)+\exp _{0}\left\{Q t / \varepsilon^{2}\right\} \psi^{1}(0, u, x, \tau) .
\end{aligned}
$$

The order of the remainder can be estimated by defining the function

$$
\varphi_{\varepsilon}^{(N)}(t, u, x, \tau)=\varphi_{\varepsilon}^{(0)}(t, u, x, \tau)+\sum_{n=1}^{N} \varepsilon^{n}\left(\varphi^{(n)}(t, u, x, \tau)+\psi^{(n)}\left(\frac{t}{\varepsilon^{2}}, u, x, \tau\right)\right) .
$$

Then it follows for the supremum norm that (see [4, [8])

$$
\left\|\varphi_{\varepsilon}(t, u, x, \tau)-\varphi_{\varepsilon}^{(N)}(t, u, x, \tau)\right\|=O\left(\varepsilon^{N+1}\right) .
$$

\section{Conclusions}

There are many new results and approaches to deal with the asymptotic expansion for perturbed equations of Markov and semi-Markov random evolutions (see [7] [10] and others). Our approach in this paper has been aimed to finding solutions of singularly perturbed equations of semi-Markov random evolutions by reducing the semi-Markov process to an equivalent Markov process consisting of three known processes. However, the resulting Markov process has a complicated continuous phase space. Upon observing that the projective and potential operators for this Markov process were obtained in [4, we apply the method of asymptotic expansion for Markov random evolutions [7]-9] to find a recursive solution.

Acknowledgments. We wish to thank an anonymous referee for a careful reading of the paper and insightful comments and remarks. 


\section{BIBLIOGRAPHY}

1. V. S. Korolyuk and V. V. Korolyuk, Stochastic Models of Systems, Kluwer Academic Publishers, 1998. MR:1753470 (2002b:60169)

2. V. S. Korolyuk and A. V. Swishchuk, Evolution of Systems in Random Media, CRC Press, Boca Raton, FL, U.S.A., 1995. MR1413300 (98g:60116)

3. I. I. Gikhman and A. V. Skorokhod, The theory of Stochastic Processes, vol. 2, Springer-Verlag, New York, 1975. MR0375463 (51:11656)

4. V. S. Korolyuk and A. F. Turbin, Markov Renewal Processes in System Reliability Problems, Naukova Dumka, Kiev, 1982. (Russian) MR695006 (85e:60095)

5. V. S. Korolyuk and A. F. Turbin, Mathematical Foundations of the State Lumping of Large Systems, Kluwer Academic Publishers, 1993. MR 1281385 (95e:60071)

6. A. B. Vasil'eva and V. F. Butuzov, Asymptotic Methods in the Theory of Singular Perturbations, Vysshaya Shkola, Moscow, 1990. (Russian) MR1108181(92i:34072)

7. S. Albeverio, V. S. Korolyuk, and I. V. Samoilenko, Asymptotic expansion of semi-Markov random evolutions, Stochastics: An International Journal of Probability and Stochastics Processes, 81 (October 2009), no. 5, 477-502. MR2569263 (2010k:60311)

8. I. V. Samoilenko, Asymptotic expansion of Markov random evolution, Ukrainian Mathematical Bulletin 3 (2006), no. 3, 394-407. MR2330681 (2008d:60098)

9. A. Pogorui, Asymptotic expansion for the distribution of a Markovian random motion, Random Operators \& Stochastic Equations 17 (2009), 189-196. MR2560865 (2010i:60217)

10. M. A. Pinsky, Lectures on Random Evolutions, World Scientific Publishing, 1991. MR1143780 (93b:60160)

Zhytomyr State Ivan Franko University, Velyka Berdychivs'Ka St. 40, Zhytomyr 10008, UKRAINE

E-mail address: pogor@zu.edu.ua

Centro de Electrónica y Telecomunicaciones, itesm, E. Garza Sada 2501 Sur, C.P. 64849, Monterrey, N.L., MÉxico

E-mail address: rmrodrig@itesm.mx

Received 20/OCT/2009

Originally published in English 